\title{
RELATIONSHIP BETWEEN TEMPORARY EMOTION OF STUDENTS AND PERFORMANCE IN LEARNING THROUGH COMPARING FACIAL EXPRESSION ANALYTICS
}

\author{
Mahima Maharjan ${ }^{1}$, Soo-Hyeong Kim², Soonja Yeom ${ }^{1}$ and Si Fan ${ }^{3}$ \\ ${ }^{1}$ School of Technology, Environment and Design, Private Bag 87 University of Tasmania, Australia \\ ${ }^{2}$ Department of Computer Science, Chonnam National University, Gwangju, South Korea \\ ${ }^{3}$ School of Education, Locked Bag 1307, University of Tasmania, Australia
}

\begin{abstract}
This paper presents a study on temporary emotion of students and their performance related to learning activities. This paper elucidates different kinds of facial expressions elicited during the activities: quiz and a movie trailer with the help of existing facial expression analyzing applications. The user's expressions are recorded as video while watching the movie trailer and doing the quiz. The video is processed by different applications which gives the score for different emotions. The results obtained are studied to find which emotion is mostly prevalent among the user in different situations. From this study, it is shown that students experience seemingly different emotions during the activity. The emotions they portrayed were confusion, sadness, anger and neutral. This study explores the use of affective computing for further comprehension of students' emotion in learning environment.
\end{abstract}

\section{KEYWORDS}

Facial expression, Emotion, Affective computing, Learning

\section{INTRODUCTION}

Affective issues in learning technologies are concerned with emotional areas such as inspiration, attitudes and feelings (Jones and Issroff 2004). Emotion is one of the most researched topics in the study of psychology (Cho and Heron 2015, Plutchik 2001). Plutchik (2001) notes that over 90 definitions of emotion have been proposed since 20th century; emotion is expressed in terms of anger, despair, joy and grief. Learning strategies like critical thinking (Cho and Heron 2015), and effort contributed less in explaining students' achievement compared to motivation and emotions related to it. Therefore, more motivational and emotional supports are necessary to enhance student's success. Moreover, affective states play a significant role in daily activities of humans, including tasks performed in front of a computer (Jones and Issroff 2005). Objective study of emotion itself is challenging task as several emotions could be experienced at the same time (Plutchik 2001). Emotion can be treated as the on/off switch for learning (Vail 1994). Intellectual energies and capacities are increased/ decreased by positive/ negative emotional states. Having to attend the time limit quiz where there is continuous tension of ongoing time and memory, can impede students' performance increasing anxiety (Jones and Issroff 2005).

LeDoux (2003) noted that the limbic system, also known as the emotional brain is one part of the three-way view of human brain interprets the emotional value of incoming stimuli and decides if they are neutral, good or deadly; controls the ability to learn, memory and make novel connections. The active states of different parts of brain gives different facial expressions. For example: anger result from activation of some parts of brain that help to react with more speed and strength while overpowering prudent things replacing cautiousness with aggressiveness and compassion with resentment. Emotional expressions are states that define different ways of thinking. Furthermore, facial signs are perceived differently than non-facial signs such as audio for the same emotions (Ekman 2003). 
Robinson (2008) categorizes interest, curiosity, surprise, joy, and love as positive emotions; while panic, aversion or disgust, fear, anger, anxiety, sorrow, and frustration are considered as negative emotions (Robinson 2008). Positive states like happiness cause positive impacts on learning while negative states like anger and sadness generate negative effects (Macfadden 2005). The theoretical assumption of control-value theory suggest that negative emotions can produce positive outcome (Pekrun 2006). To the contrary, "learning by positive reinforcement" does not account for so much of how people learn.

The relationship between the emotional intelligence (EI) and academic performance (AP) of the students shows substantial positive association between them. EI is ability to perceive and express emotion. It is used to facilitate thought, understand and reason with emotion (Mayer et al. 2001). The technologies which recognize and express emotional states are leading to the development of different algorithms which can provide artificial environment to explore human nature further, control cognition, attention and action. Until now, to enable computers to adapt the human behavior has been the main goal rather than the way round (Minsky 2013).

There have been only limited studies about affective and emotional outcome associated learning. Most of the studies are only focused on positive emotions such as happy portraying positive outcome or success in the overall grade (Macfadden 2005, Fitriani, Apriliaswati and Rosnija 2017); yet the evidence of negative emotion producing success is somewhat limited. In the present study, we would like to investigate if there is correlation between negative emotions and positive outcome.

\section{LITERATURE REVIEW}

Many facial expressions correspond to emotions. The most commonly used system of facial action analysis was developed by Ekman and Friesan known as Facial Action Coding System (FACS) (Ekman 2003), which determines facial muscular movement based on a set of 46 facial Action Units (AUs). These AUs are automatically related to the contraction of a specific set of facial muscles. For instance, AU0 represents neutral face, AU1 represents inner brow raiser and so on. The six basic emotions: happy, sad, angry, surprised, scared, disgusted, plus neutral are a combination of one or many AUs. FACS intensities are represented by letters A-E (described as maximum, severe or extreme, marked or pronounced, slight and trace respectively), with A being minimal and $\mathrm{E}$ as maximal intensity to the action unit number.

Faces contain details about the identity, mood and mind state which are the mechanisms governing the emotions and the most expressive way humans portray emotions is through facial expressions (Sathik and Jonathan 2013). Facial expressions are signaled by different muscle, eye and lip movements (Ekman 2003). Positive facial expressions are indicated by Eyes Opening wide with raising the eye brows which are represented as comprehension. Negative emotions are conveyed by contracting eyes and eye brows raised high, enlarged eyes, curling lips which represent student's incomprehension and confused state (Sathik and Jonathan 2013). Some of the common emotions during the learning process are delight, hope, pride, satisfaction, anger, shame, frustration and boredom. Positive emotions like enjoy, hope associate motivation, effort and learning strategies (Pekrun et al. 2011). Positive affect also increases motivation in learners (Goleman 2006). Negative emotions impact students' performance such as inactive behavior, less concentration, motivation and confidence (Fitriani, Apriliaswati and Rosnija 2017). Emotions upset mental life and those who have negative emotions like anxiety, annoyance and depression are not capable of learning (Goleman 2006). In contrast to conventional classrooms, where lecturers for the most part give direction and structure (both verbal and non-verbal direction), students should exercise self-administrative ability to achieve their learning objectives in today's context (Artino 2010). For example: a person may feel worry or anxiety due to threat of an encounter. Failure leads people to convey negative emotions which as a result outcomes low self-esteem (Schunk 1996). Self-confidence is factor in learning and help to show more perseverance when problems arise. Some researchers also claim that joy and anxiety experienced by the students depends on individual goals (Goleman 2006). If the goal of the student is achieving high marks and if the goal is approaching, the student might feel positive emotions like joy; whereas if the students is not satisfied, the student might experience negative emotions like anxiety (Pekrun 2006). Other research claims that success instigates positive emotions while failure promotes negative emotions (Weiner 1985). Negative states like confusion could prove beneficial because of increased attention on learning activities which consequently gives higher performances (D'mello et al. 2014). Moreover, negative emotion like anxiety is induced if a student expects failure in the quiz or 
examination, whereas no anxiety will occur if student does not care about it or does not expect failure. Furthermore, Stress which is also a negative state of emotion is considered to encourage or inhibit learning process (Vogel and Schwabe 2016). Surprise is a strange situation which refers an inconsistency among prior and new information (Sathik and Jonathan 2013). While negative emotions are associated with low performance; there is limited research on negative emotions portraying positive outcome. The need for more precise understanding of relation between facial emotion and learning outcome is being driven today by technologies being built to interact with learners.

In this research, four different applications are investigated for analysis of the student's emotions which are explained as below.

OpenFace: It is intended for computer vision and machine learning researchers, affective computing community and people interested in building interactive applications. In this application, facial behavior is composed of facial landmark detection, head pose estimation, facial AU recognition, and eye-gaze estimation (Baltrusaitis, Robinson and Morency 2016).

Noldus Facereader: It has an accuracy rate of 88\% (Lewinski, Den Uyl and Butler 2014); allows evaluation of expressions quantitatively. Viola Jones cascaded algorithm with deep face classification for finding the position of the face in an image. Active Appearance Model (AAM) is used to synthesize an artificial model. The expressions are represented as the scale between 0 to 1 . The trained artificial neural network is used to classify the six basic or universal emotions (Ekman 2003). Facereader can analyze 20 AUs and 3 affective attitudes (interest, boredom and confusion).

Face Plus Plus: It only supports image. The application analyzes and identifies emotion of detected faces and provides simple and powerful APIs and SDKs to add into deep learning-based image analysis recognition technology (Face++ 2019).

Affectiva: It uses cross platform real-time multi-phase expression recognition toolkit, for research in Emotion AI. Affectiva detects 14 facial actions and 7 different emotional states and has an accuracy of $84.27 \%$ and tested for robust databases (Lisetti and Schiano 2000).

Based on the empirical findings of the technologies, our approach is to experiment and get results from various technologies dealing with affect and facial emotions to better comprehend the emotions in learning.

\section{MOTIVATION}

This paper studies the gap between the students learning and their related emotions. Faces broadcast information about not only emotion but also mood, attitude, character, intelligence, attractiveness, age, sex, race and so on (Ekman 2003). Emotion messages conveyed by facial signals is the primary focus of this study. As mentioned earlier, emotions refer to feelings such as fear, anger, happiness, surprise, confusion etc.; when these feelings occur, they are visible in face in form of facial muscles contractions (Ekman and Keltner 1970). Thus, emotion can be judged from facial signals (Ekman and Friesen 2003). The main significance of this study is to determine if the facial expression correspond to recognized emotions and to what degree do facial emotions predict student accomplishment. This study has been carried to find the correlation between positive as well as negative emotion in learning outcomes. However, this study does not emphasize on any one of those emotions. This paper would help Human Computer Interaction (HCI), Artificial Intelligence (AI) and affective studies understand and respond to the information provided by the face.

\section{METHODOLOGY}

This section describes the data collection and cleaning to achieve noise free results. An informed consent was obtained from all the participants for the data collection and storage. In this research, the data is collected in the form of video for quiz activity and the movie trailer, which is then analyzed with the help of application described in Section 2. Depending upon the applications, expressions and their understandings may vary. Facereader is commercially trusted and reliable indicator of facial expressions of basic emotions in terms of factors like facial expressions, facial states, AUs, affective attitudes, heart rate and many more. Thus, more analysis is done from Facereader and most of the results are focused based on Facereader. After the analysis, the result of relationship between emotion and the student learning is discussed. Computer-oriented activities 
generally generates neutral expressions; hence, to study sparse human emotion, movie trailer is used. Since movie trailer comprises of different scenes (action, humor, sad, surprise, fear and so on), we used movie trailer data to study different emotions. Students who are enrolled in one of the Master units are requested to participate in the study by recording their faces during the activities. The participation is voluntary and could be withdrawn anytime without an explanation. A total of 7 students of University of Tasmania, of which 33\% of the participants were male and $67 \%$ female participated in the research. The participants were mainly international students; among the seven participants, 4 of the participants were from China, the other three were from Hong Kong, Korea and Nepal, aged between 20-30 years. Only those who were enrolled for the summer course are included in the analysis. The University cloud storage is used to store the video recordings from the participants, expression, affective intensity and AUs are recorded during the evaluation. The result obtained from applications are discontinuous; so, it has been resampled to make it continuous. The result of the analysis is then compared with the participant's grade of the quiz as well as overall performance in the unit.

\section{EXPERIMENT AND RESULTS}

The experiment is performed based on the video sample recorded as mentioned in Section 4. The analysis is broken into three sections: expression, affective attitude and AU intensity analysis.

\subsection{Expression Intensity Analysis}

The same dataset is used for all the application as mentioned in Section 2 to analyze and compare the effect of participants basic emotions towards the quiz and a movie trailer.

\subsubsection{Facereader Results}

Among seven participants studied, one of the results is illustrated in Figure 1, which depicts emotions score $(0-1)$ in Y-axis and time (seconds) in X-axis. It is seen that there is a significant effect of participants' neutral, sad and angry emotion throughout the quiz. The other emotions: happy, scared and disgusted are negligible. The highest score is seen for sad emotion. This indicates that participant might have faced difficult questions or unseen questions which participants found tough to answer. Because of difficult questions, the participants' consequence to angry emotion is caused (Macfadden 2005). The neutral emotion could be because the participants are thought to emote to computers and are aware of the video being recorded and therefore the notable facial emotions is rarely shown. Therefore, emotions like fear and surprise are kept away from computers. Techno-phobic people could also experience anger, fear towards computers (Lisetti and Schiano 2000). Ambivalent could be assumed as an impact of happiness, wellbeing and satisfaction. The result of neutral facial expression could infer the ambivalent state (Pekrun 2006). The high score of sad during the quiz indicated that participant might have faced difficult question. As stated by Vogel and Schwabe (2016), negative emotional states like stress represented by sad and angry facial expressions brings positive learning process and memory as well (Vogel and Schwabe 2016).

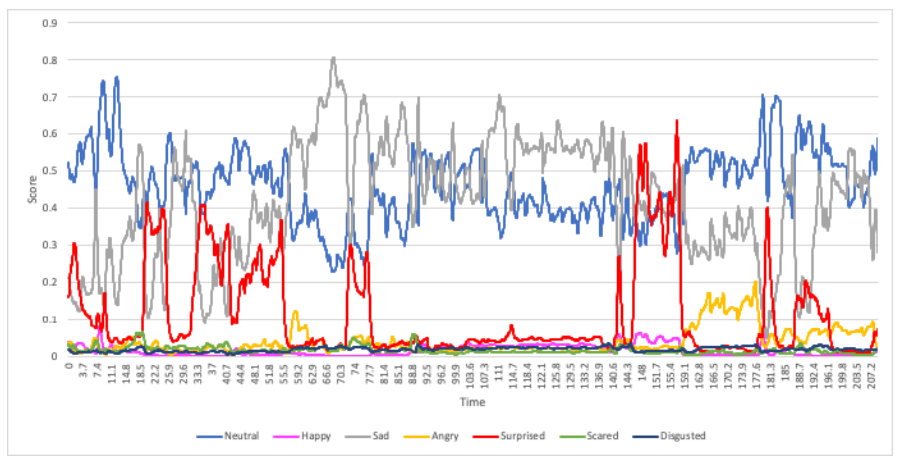

Figure 1. Emotions at quiz by Facereader 
Scores for other emotions changed regularly but remained at lower levels. Surprise is a strange situation which refers an inconsistency among prior and new information (Sathik and Jonathan 2013). Thus, surprise emotion could be because of difficult or never seen questions at the beginning of the video. It could also imply that the participant is amazed of the result. In addition, the score for angry with surprise emotion are observed at the end of the quiz. It depicts that the participant is angry as well as astounded during the difficult or unseen question. Delight and surprise are relatively low compared to confusion, boredom and frustration in the complex learning process (D’mello et al. 2014).

In summary, the participant experienced different positive emotions: surprised and happy as well as negative emotions: sad, disgusted and neutral as seen in the Figure 1 and Table 1.

Table 1. The average score of emotions experienced by Participants during the quiz and movie trailer

\begin{tabular}{lll}
\hline Expressions & \multicolumn{2}{c}{ Activities } \\
\cline { 2 - 3 } & Quiz & Movie trailer \\
Neutral & $\mathbf{0 . 6 4 4 9 1 4}$ & $\mathbf{0 . 6 7 5 5 9 5}$ \\
Happy & 0.01874 & $\mathbf{0 . 1 3 9 5 3 2}$ \\
Sad & $\mathbf{0 . 1 6 1 1 0 4}$ & 0.073182 \\
Angry & 0.070149 & 0.014673 \\
Surprised & 0.046198 & 0.015427 \\
Scared & 0.034932 & 0.019614 \\
Disgusted & 0.010972 & 0.022628 \\
\hline
\end{tabular}

\subsubsection{Face Plus Plus Results}

The result obtained from Face Plus Plus insinuates that neutral is dominant expression (Figure 2 (a)) which resembles the result given by the Facereader. The negative emotions like sadness or anger are prominent in the quiz similar to the result demonstrated in Figure 1 and Table 1.

\subsubsection{Affectiva Results}

The result obtained as demonstrated in Figure 2(b) and Figure 2(c) indicates that disgust expression is dominant over other expressions which is quite different from the other two applications. The other notable emotions are fear and sadness which however matches the result from the Facereader and Face Plus Plus results. The possible reason of variant result could be because of the sensitivity of the mobile phone camera and distance between the camera and data. The result obtained which are disgust, fear and anger could be compared to neutral expressions (Sprengelmeyer et al. 1998).

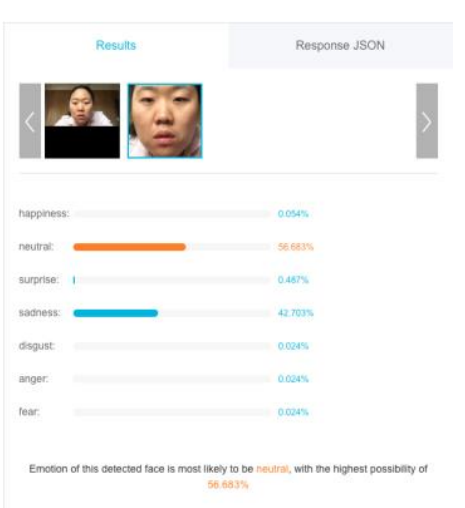

Figure 2 (a). Realtime facial emotion detection by Face Plus Plus

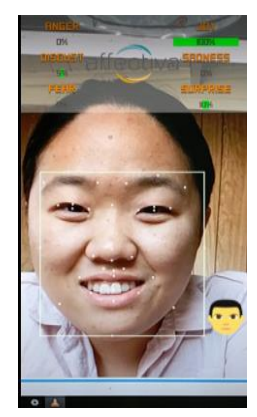

b) Expressions in Affectiva identified as joy

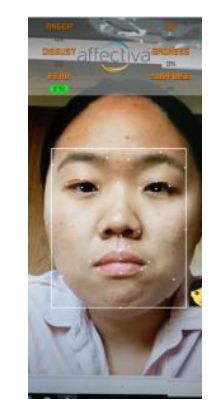

c) Expressions in Affectiva identified as fear

\subsection{Affective Attitude Intensity Analysis}

Affective attitudes are complex emotional states that differ from the basic emotions (Ekman 2003). Affective attitudes are associated with a mixture of AUs activations over a time. By examining the participants' responses, a clear distinction of affective intensities like confusion, boredom and interest are found. Interest is 
induced from occurrence of new incident, intricate and unacquainted and as logical (Silvia 2009). The research also points out that interest is related to physiological activation, behavioral exploration and novelty. As mentioned in Section 1 and Section 2, the emotions such as confusion represents the positive impact on learning because of increased focus (D'mello et al. 2014). Learning comes from confusion, problems, taking risks and discomfort. Learners need to cope with uncertainty which result in negative emotions. Nevertheless, confusion is somewhat made for concentration, worry and skepticism (Rozen and Cohen 2003). In this study, level of confusion as well as interest is observed high in the quiz. Regarding the movie trailer, boredom was more prevalent.

\subsection{Action Unit Intensity Analysis}

Results from two applications: Openface and Facereader suggest that AU for inner brow raiser are high compared to other AUs. In addition to inner brow, outer brow raiser is also prevalent in Facereader. The action of opening the eyes wider and lifting the eyebrows bear positive emotions; whereas, negative emotions are represented by shrinking eyes with lowering eyebrows and wrinkles on forehead. The raised eyebrows enlarged eyes and curled lips convey negative emotions (Sathik and Jonathan 2013). These negative emotions are the indications of incomprehension and confusion. In this study, lip corner depressor and chin raiser were also detected, in addition to eyebrows movement, which are the indications of negative emotions. The AUs (X-axis) and frequencies of five intensities (Y-axis) of AUs of Facereader are shown in Figure 3.

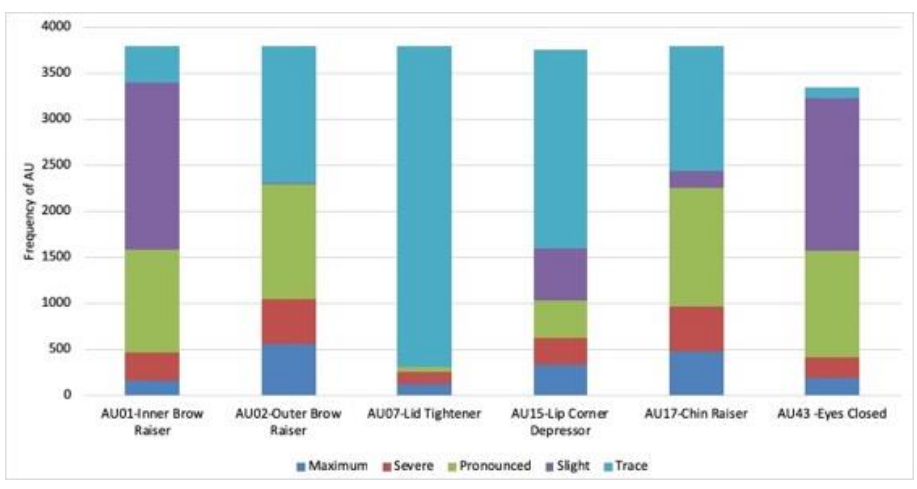

Figure 3. Notable AUs during the quiz by Facereader

\section{DISCUSSION}

The present study investigated the emotions in learning activities of students. As specified in the Section 1 and Section 2, there is now an agreement and clashed finding on the theory that positive emotions cause positive impact and negative emotions cause negative impact. This study is a slight contribution to the discussion, adding one more voice that suggests, positive emotions or facial expressions do not necessarily give positive outcome. Emotions such as confusion represent the positive impact on learning as a result of increased focus (D'mello et al. 2014). By collating the result of facial expressions and written responses, it is found that learning process brings negative emotions like sad and angry. Another finding of our study is that facial expressions partially corresponded to the result mentioned by the participant. This suggests that facial expressions may not be the only one factor indicating the emotions. Affective attitudes like confusion, boredom and interested is not included but is one of the factors affecting the learning process (Ekman 2003). In the context of learning, higher level of confusion contributes frustration may produce positive outcomes (Rozin and Cohen 2003) which occurred concurrently with negative emotions like angry, sad and neutral. Yet, the paper does not encourage negative emotions while in learning process. Nevertheless, the negative emotions like confusion and worry contributes positive outcome is seen in our study. This has been matched with participant self-reports result and grades of the participants. 
The facial actions reported as the most common in confusion involve the eyes and eyebrows: narrowing of the eyes, lowering and bringing together of the eyebrows. These actions, often characteristic of frowns, are seen in several facial expressions. These situations may be related to confusion (Ekman 2003). Results from facial expression study of the movie trailer support the theory of positive emotions such as happiness is directly proportional to outcome. Positive emotions strengthen inspiration while negative can be disadvantageous to learning process. Finally, boredom is also prevalently seen in the movie trailer. As the previous researches regarding the boredom emotion say that boredom is induced from absence of positive and negative emotions (Ekman 2003), the result achieved from the analysis may be because of already watched movie trailer.

\section{CONCLUSION AND FUTURE WORK}

As mentioned in Section 2, much researches has been done to understand the emotional expressions conveyed by learners. In this research paper, the results of different facial expression recognition applications are studied by recording video of participant's facial expression performing quiz activity and watching a movie trailer. The result shows variant neutral, sad, surprise and disgust emotions during the quiz. Negative emotions like anger, sadness and affective states like confusion has extensive consequences on students learning process. The outcome from the study of the movie trailer is more of a positive facial expression like happy and surprise. The outcome studied are compared to the self-reports and grades of the participants. These reports accompanied the plausible emotions generated by the studied applications. The result of our study might be helpful to enhance the learning setting by comprehending how students react to the learning environment.

The personal attributes and personality of the individuals across the time are not studied. It is assumed that the students who studied the unit with the positive attitude likely maintained high score. The variation of emotions is slightly biased because students are pre-informed about quiz and the movie trailer is old, so they might have already watched that video. It is also reasonable that the graduate students are already motivated to study as participants are in higher level of studies.

Future work will focus on the verification of result from the applications used and determine whether disgust emotion relate to negative emotions like anger and sadness. As the number of participants were low in the research, more participants will be researched in the future. Undeniably, there has been plenty of studies in neuroscience and psychology about the significance of emotional intelligence in rational decision making, performance and adaptation; the aim would be to better comprehend human emotions by building tools with increased awareness of the users' expressions and mental states which would support psychologists to understand more complex processes of human systems with different ethnicity, gender and ages. Besides, as most of the facial expressions are brief, they are missed by observers as well as computers. These micro expressions usually reveal the real emotions a person is trying to conceal, such as delight, hope, pride, satisfaction, anger, shame, frustration and boredom. Automatic recognition procedures need to be studied further.

\section{ACKNOWLEDGEMENT}

The research involving human subjects were approved by the Social Science Human Research Ethics Committee (HREC) Tasmania Network (reference H0016953).

\section{REFERENCES}

Artino Jr, A.R., 2010. Online or face-to-face learning? Exploring the personal factors that predict students' choice of instructional format. The Internet and Higher Education, Vol. 13, No. 4, pp.272-276.

Baltrušaitis, T., Robinson, P. and Morency, L.P., 2016, March. Openface: an open source facial behavior analysis toolkit. In 2016 IEEE Winter Conference on Applications of Computer Vision (WACV), pp. 1-10, IEEE.

Cho, M.H. and Heron, M.L., 2015. Self-regulated learning: the role of motivation, emotion, and use of learning strategies in students' learning experiences in a self-paced online mathematics course. Distance Education, Vol. 36, No.1, pp.80-99. 
D’Mello, S., Lehman, B., Pekrun, R. and Graesser, A., 2014. Confusion can be beneficial for learning. Learning and Instruction, Vol. 29, pp.153-170.

Ekman, P., 2007. Emotions revealed: Recognizing faces and feelings to improve communication and emotional life. Macmillan.

Face++, 2019, viewed December 24 2018, <https://www.faceplusplus.com>

Goleman, D., 2006. The socially intelligent. Educational leadership, Vol. 64, No. 1, pp.76-81.

Fitriani, I., Apriliaswati, R. and Rosnija, E., Analysis of EFL Students'negative Emotions Towards English Learning Process in SMPN 23 Pontianak. Jurnal Pendidikan dan Pembelajaran, Vol. 6, NO. 6.

Jones, A. and Issroff, K., 2005. Learning technologies: Affective and social issues in computer-supported collaborative learning. Computers \& Education, Vol.44, No. 4, pp.395-408.

LeDoux, J., 2003. The emotional brain, fear, and the amygdala. Cellular and molecular neurobiology, Vol. 23, No. 4-5, pp.727-738.

Lewinski, P., den Uyl, T.M. and Butler, C., 2014. Automated facial coding: Validation of basic emotions and FACS AUs in FaceReader. Journal of Neuroscience, Psychology, and Economics, Vol. 7, No. 4, p.227.

Lisetti, C.L. and Schiano, D.J., 2000. Automatic facial expression interpretation: Where human-computer interaction, artificial intelligence and cognitive science intersect. Pragmatics \& cognition, Vol. 8, No. 1, pp.185-235.

MacFadden, R.J., 2005. Web-based education in the human services: Models, methods, and best practices, Vol. 23. Psychology Press.

Magdin, M. and Prikler, F., 2018. Real Time Facial Expression Recognition Using Webcam and SDK Affectiva. International Journal of Interactive Multimedia \& Artificial Intelligence, Vol. 5, No. 1.

MAVER, J.D., SALOVEV, P., Caruso, D.R. and Sitarenios, G., 2001. Emotional intelligence as a standard intelligence. Emotion, Vol. 3, No. 1, pp.232-242.

Minsky, M., 2007. The emotion machine: Commonsense thinking, artificial intelligence, and the future of the human mind. Simon and Schuster.

Oudeyer, P.Y., Gottlieb, J. and Lopes, M., 2016. Intrinsic motivation, curiosity, and learning: Theory and applications in educational technologies. In Progress in brain research, Vol. 229, pp. 257-284, Elsevier.

Pekrun, R., 2006. The control-value theory of achievement emotions: Assumptions, corollaries, and implications for educational research and practice. Educational psychology review, Vol. 18, No. 4, pp.315-341.

Pekrun, Reinhard, et al. "Measuring emotions in students' learning and performance: The Achievement Emotions Questionnaire (AEQ)." Contemporary educational psychology 36.1 (2011): pp. 36-48.

Plutchik, R., 2001. The nature of emotions: Human emotions have deep evolutionary roots, a fact that may explain their complexity and provide tools for clinical practice. American scientist, Vol. 89, No. 4, pp.344-350.

Robinson, J., 2008. Music and emotions. Journal of Literary Theory, Vol. 1, No. 2, pp.395-419.

Rozin, P. and Cohen, A.B., 2003. High frequency of facial expressions corresponding to confusion, concentration, and worry in an analysis of naturally occurring facial expressions of Americans. Emotion, Vol. 3, No. 1, p.68.

Sathik, M. and Jonathan, S.G., 2013. Effect of facial expressions on student's comprehension recognition in virtual educational environments. SpringerPlus, Vol. 2, No. 1, p.455.

Schunk, D.H., 1996. Attributions and the Development of Self-Regulatory Competence.

Silvia, P.J., 2009. Looking past pleasure: anger, confusion, disgust, pride, surprise, and other unusual aesthetic emotions. Psychology of Aesthetics, Creativity, and the Arts, Vol. 3 No. 1, p.48.

Sprengelmeyer, R., Rausch, M., Eysel, U.T. and Przuntek, H., 1998. Neural structures associated with recognition of facial expressions of basic emotions. Proceedings of the Royal Society of London. Series B: Biological Sciences, Vol. 265, No. 1409, pp.1927-1931.

Vail, P.L., 1994. Emotion: The on/off switch for learning. Modern Learning Press.

Vogel, S. and Schwabe, L., 2016. Learning and memory under stress: implications for the classroom. npj Science of Learning, Vol. 1, p.16011.

Weiner, B., 1985. An attributional theory of achievement motivation and emotion. Psychological review, vol. 92, No. 4, p.548. 